\title{
ON THE RATE OF CONVERGENCE OF THE IMAGE SPACE RECONSTRUCTION ALGORITHM
}

\author{
JiAn Han, Lixing Han* ${ }^{*}$, Michael NeUmanN ${ }^{* *}$ AND UPENDRA PRASAD
}

Abstract. The Image Space Reconstruction Algorithm (ISRA) of Daube-Witherspoon and Muehllehner is a multiplicative algorithm for solving nonnegative least squares problems. Eggermont has proved the global convergence of this algorithm. In this paper, we analyze its rate of convergence. We show that if at the minimum the strict complementarity condition is satisfied and the reduced Hessian matrix is positive definite, then the ISRA algorithm which converges to it does so at a linear rate of convergence. If, however, the ISRA algorithm converges to a minimum which does not satisfy the strict complementarity condition, then the rate of convergence of the algorithm can degenerate to being sublinear. Our results here therefor hold under more general assumptions than in the work of Archer and Titterington who assume that at a minimum point all Lagrange multipliers are zero.

We provide numerical examples to illustrate our rate of convergence results and to explain why the ISRA algorithm usually appears to converge slowly. Our work here heuristically justifies why the Lee-Seung algorithm for solving nonnegative matrix factorization problems has a slow rate of convergence.

Mathematics subject classification (2000): 65F20, 65Y20, 15A48, 15A23.

Keywords and phrases: nonnegative least squares, ISRA (Image Space Reconstruction Algorithm), rate of convergence, nonnegative matrix factorization.

\section{REFERENCES}

[1] B. Anconelli, M. Bertero, P. Boccacci, M. CARbiller, And H. LANTERI, Iteative methods for the reconstruction of astronomical images with high dynamic range, Journal of Computational and Applied Mathematics, 198 (2007), 321-331.

[2] J. M. M. Anderson, C. K. Yust, And B. A. MaIR, Minimum distance methods for transmission tomography, 1996 IEEE Nuclear Science Symposium, Vol. 3 (1996), 1767-1771.

[3] G. E. B. ARCHER AND D. M. TITTERINGTON, The Image Space Reconstruction Algorithm (ISRA) as an alternative to the EM algorithm for solving positive linear inverse problems, Statistica Sinica, 5 (1995), 77-96.

[4] S. Bellavia, M. MacConi, AND B. Morini, An interior point Newton-like method for non-negative least squares problems with degenerate solution, Numerical Linear Algebra with Applications, Vol. 13, No. 10 (2006), 825-846.

[5] A. Berman AND R. J. Plemmons, Nonnegative Matrices in the Mathematical Sciences, SIAM, 1994.

[6] M. Berry, M. Browne, A. LangVille, P. PauCA, And R. J. Plemmons, Algorithms and applications for approximate nonnegative matrix factorization, Computational Statistics and Data Analysis, 52 (2007), 155-173.

[7] M. BERTERO AND P. BOCCACCI, Image restoration methods for the Large Binocular Telescope (LBT), Astronomy \& Astrophysics Supplementary Series, 147 (2000), 323-333.

[8] A. CICHOCKI, R. ZDUNEK, AND S. AMARI, Nonnegative matrix and tensor factorization, Signal Processing Magazine, IEEE, Vol. 25, No. 1 (2008), 142-145.

[9] M. E. DAUBE-WiTHERSPOON AND G. MUEHLLEHNER, An iterative image space reconstruction algorithm suitable for volume ECT, IEEE Trans. Med. Imaging, MI-5 (1986), 61-66. 
[10] A. R. DE PIERRO, On the convergence of the iterative image space reconstruction algorithm for volume ECT, IEEE Trans. Med. Imaging, MI-6 (1987), 174-175.

[11] P. P. B. EGGERMONT, Multiplicative iterative algorithms for convex programming, Linear Algebra and its Applications, 130 (1990), 25-42.

[12] L. ElSNER, I. KOLTRACHT, AND M. NEUMANN, On the convergence of asynchronous paracontractions with application to tomographic reconstruction from incomplete data, Linear Algebra and its Applications, 130 (1990), 65-82.

[13] R. Fletcher, Practical Methods of Optimization, Second edition, John Wiley \& Sons, 1987.

[14] E. F. GONZALEZ AND Y. ZHANG, Accelerating the Lee-Seung algorithm for nonnegative matrix factorization, Technical Report TR05-02, Department of Computational and Applied Mathematics, Rice University, Houston, TX 77005, 2005

[15] S. Holte, P. SCHMIDLIN, A. LindEN, G. ROSENQVIST, AND L. ERIKSSON, Iterative image reconstruction forpositive emission tomography: A study of convergence and quantitation problems, IEEE Transactions on Nuclear Science, Vol. 37, No. 2 (1990), 629-635.

[16] R. A. HoRn AND C. R. Johnson, Matrix Analysis, Cambridge University Press, 1985.

[17] L. KAUFMAN, Maximum likelihood, least squares, and the penalized least squares for PET, IEEE Transactions on Medical Imaging, 12 (1993), 200-214.

[18] D. KIM, S. SRA, AND I. DHILLON, Fast Newton-type methods for the least squares non-negative matrix approximation problem, Proceedings of SIAM Conference on Data Mining, 2007.

[19] C. J. LiN, Projected gradient methods for nonnegative matrix factorization, Neural Computation, 19 (2007), 2756-2779.

[20] D. D. LEE AND H. S. SEUNG, Learning the parts of the objects by non-negative matrix factorization, Nature, 401 (1999), 788-791.

[21] D. D. LEE AND H. S. SEUNG, Algorithms for non-negative matrix factorization, Advances in Neural Information Processing Systems 13, 2000.

[22] G. KONTAXAKIS, L. G. STRAUSS, AND G. VAN KAICK, Optimized image reconstruction for emission tomography using ordered subsets, median root prior and a web-based interface, 1998 IEEE Nuclear Science Symposium, Vol. 2 (1998), 1347-1352.

[23] G. Kontaxakis, L. Strauss, T. Thiereou, and M. J. Ledesma-Carbayo, A. Santos, A. A. PAVLOPOULOS, AND A. DIMITRAKOPOULOU-STRAUSS, Iterative image reconstruction for clinical PET using oedered-subsets, median root prior, and a web-based interface, Molecular Imaging and Biology, Vol. 4, No. 3 (2002), 219-231.

[24] J. Morales, N. Medero, N. G. SANTIAGO, AND J. Sosa, Hardware Implementation of Image Reconstruction Algorithm using FPGAs, MWSCAS '06. 49th IEEE International Midwest Symposium on Circuits and Systems, Vol. 1 (2006), 433-436. 2006.

[25] J. Nocedal AND S. WRIGHT, Numerical Optimization, Springer, 1999.

[26] J. M. OlLINGER AND J. S. KARP, An evaluation of three algorithms for reconstructing images from data with missing projections, IEEE Transactions on Nuclear Science, Vol. 35, No. 1 (1988), 629-634.

[27] L. A. SHEPP AND Y. VARDI, Maximum likelihood reconstruction in emission tomography, IEEE Trans. Med. Imaging, MI-1 (1982), 113-122.

[28] Y. VARDI, L. A. SHEPP, AND L. KAUFMAN, A statistical model for positron emission tomography, J. Amer. Statist. Assoc. 80 (1985), 8-38.

[29] M. Velez-Reyes, A. Puetz, P. Hoke, R. B. Lockwood, AND S. Rosario, Iterative Algorithms for unmixing hyperspectral imagery, Proceedings of IPIE, Vol. 5093 (2003), 418-429.

[30] R. VIO, J. NAGY, L. TENORIO, AND W. WAMSTEKER, A simple but efficient algorithm for multiple-image deblurring, Astronomy \& Astrophysics, 416 (2004), 403-410.

[31] C. R. VoGEL, Computational Methods for Inverse Problems, SIAM, Philadelphia, 2002. 